\title{
The Optimization of a 4-Element Input Lens on a Hemispherical Deflector Analyzer Using SIMION
}

\author{
TJM Zouros ${ }^{1,2}$, A. Kanellakopoulos ${ }^{3}$, I. Madesis ${ }^{1,2}$, A. Dimitriou ${ }^{1,2}$, M. Fernández-Martín ${ }^{4}$, \\ G. Martínez ${ }^{4}$ and T.J. Mertzimekis ${ }^{3}$ \\ ${ }^{1 .}$ Dept. of Physics, Univ. of Crete, P.O Box 2208, GR 71003 Heraklion, Greece. \\ 2. Tandem Accelerator Laboratory, INPP, NCSR Demokritos, GR 15310 Ag Paraskevi, Greece. \\ 3. Dept. of Physics, Univ. of Athens, Zografou Campus, GR 15784 Athens, Greece. \\ 4. Dept. de Física Aplicada III, Fac. de Física, UCM 28040-Madrid, Spain.
}

We report on our simulation procedure for finding the optimal voltages of an input lens used for high resolution Auger projectile electron spectroscopy at the new atomic physics experimental station located at the $5 \mathrm{MV}$ Tandem Accelerator of the INPP at NCSR "Demokritos" [1]. The station consists of a hemispherical deflector analyzer (HDA) with a 4-element injection lens and 2-D position sensitive detector (PSD) to be used for studies of projectile excitation processes in energetic ion-atom collisions. The optimization was performed by simulations using the SIMION 8.1 package [2].

Figure 1 shows our experimental setup. The HDA uses a wide gap inter-electrode distance with $R_{1}=72.4 \mathrm{~mm}, R_{2}=130.8 \mathrm{~mm}$ and a mean radius of $\bar{R}=101.6 \mathrm{~mm}$. The lens entry is paracentric and placed at $R_{0}=82.55 \mathrm{~mm}$. Details on the lens geometry can be found in [3]. The position of the detector can be controlled and in this study was placed at a distance $h=17.56 \mathrm{~mm}$ from the HDA exit.

From the analysis of the general trajectory equation of an ideal HDA, the voltages of the inner and outer hemispheres must satisfy the following equation for a reference (central) electron beam of initial kinetic energy $E_{s 0}[4]$ :

$$
q V_{R i}=\frac{E_{s 0}}{F}\left\{F-\gamma\left(\frac{R_{0}}{R_{\pi}}\right)\left[\frac{R_{0}+R_{\pi}}{R_{i}}-1\right]\right\} \quad(i=1,2)
$$

where $F$ is the pre-retardation factor and $q$ is the charge of the electron $q=-|e|$. The parameter $\gamma$ is an independent variable which sets the entry bias $V\left(R_{0}\right)$ [5], the potential acting on the central ray upon entry. The plate electrode voltage $V_{p}$ is responsible for the final electron deceleration.

SIMION uses the finite difference method to solve the Laplace equation for the potential in the region of interest. Simple initial electron distributions were "flown" into the lens entry aperture, through the HDA and finally "splatted" on the PSD. The two lens voltages VL4 and VL5 (see Figure 1) were treated as independent search parameters and varied in small steps over the entire practical voltage space for each "fly", while the beam trace width, $\Delta x_{\text {span }}$, along the dispersion direction at the PSD was recorded. The simulations were carried out for various pre-retardation factors $F$. Figure 2 shows the maps generated for all possible voltage (positive or negative) combinations for $F=1-10$ based on the minimization of $\Delta x_{\text {span }}$. The white dots indicate the voltage combinations with the 40 smallest values of $\Delta x_{\text {span }}$. The black dots represent previously experimentally determined working points [3]. Our goal was to try to improve the experimentally determined values through simulation and further improve the experimental 
setup energy resolution. In Figure 2, we observe that as the value of $F$ increases most of the optimal voltages seem to be located in the negative quadrant, which corresponds to electron deceleration.

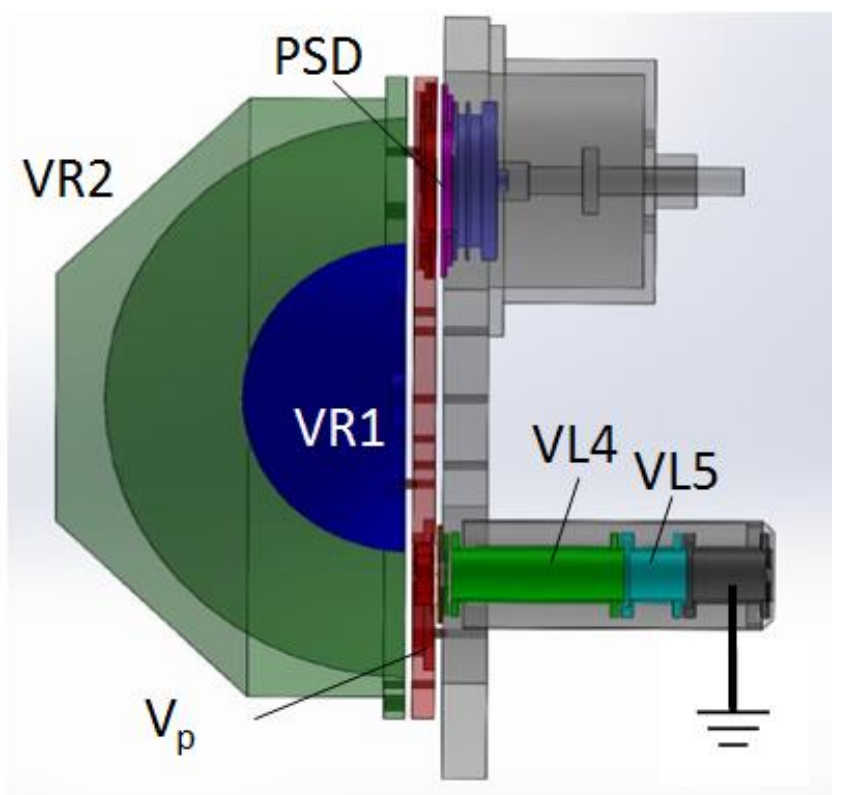

Figure 1. Geometry representation of the injection lens-HDA-PSD system. The two lens electrodes whose voltages are optimized are shown in light blue (VL5) and light green (VLA).

In order to characterize the resolution of the system, beam spots from about 8000 trajectories were computed for the nominal energy $E_{s 0}=1000 \mathrm{eV}$ and for energies differing in $\pm 10 \mathrm{eV}$ from this value. Figure 3 shows the beam spots obtained for one of the optimal sets computed with $F=1, V L 4=-697.2 \mathrm{~V}$ and $V L 5=-655.9 \mathrm{~V}$. We observe a clear separation of the spots along the dispersion direction of the analyser ( $x$-axis). The beam spots are also seen to be increasingly elongated along the $y$-direction as the energy increases.

From these distributions we determined the distance between centres to be $\approx 1.53 \mathrm{~mm}$, while the full width at the half maximum (FWHM) of the central spot was found to be $\Delta x_{p s d}=0.315 \mathrm{~mm}$. From these numbers, the energy dispersion can be readily computed for $E_{\mathrm{s} 0}=1000 \mathrm{eV}$ as:

$$
D \equiv E_{s 0} \frac{\partial x}{\partial E}=E_{s 0} \frac{1.53 \mathrm{~mm}}{10 \mathrm{eV}}=153 \mathrm{~mm}
$$

and the energy resolution is computed to be

$$
\mathcal{R} \equiv \frac{\Delta E_{\mathrm{FWHM}}}{E_{s 0}}=\frac{(0.315 \times 10 / 1.53)}{1000}=2.06 \times 10^{-3}=0.206 \%
$$

This procedure was repeated for the calculation of $D$ and $\mathcal{R}$ for all values of $F=1-8$. As $F$ increases, the separation between the central spot and those $\pm \Delta E_{s 0}$ away were also found to increase indicating 
improvement in the resolution. In Table 1 we list the optimal values of the lens voltage giving minimum $\Delta x_{\mathrm{psd}}$ in each case, together with the corresponding dispersion and FWHM resolution.
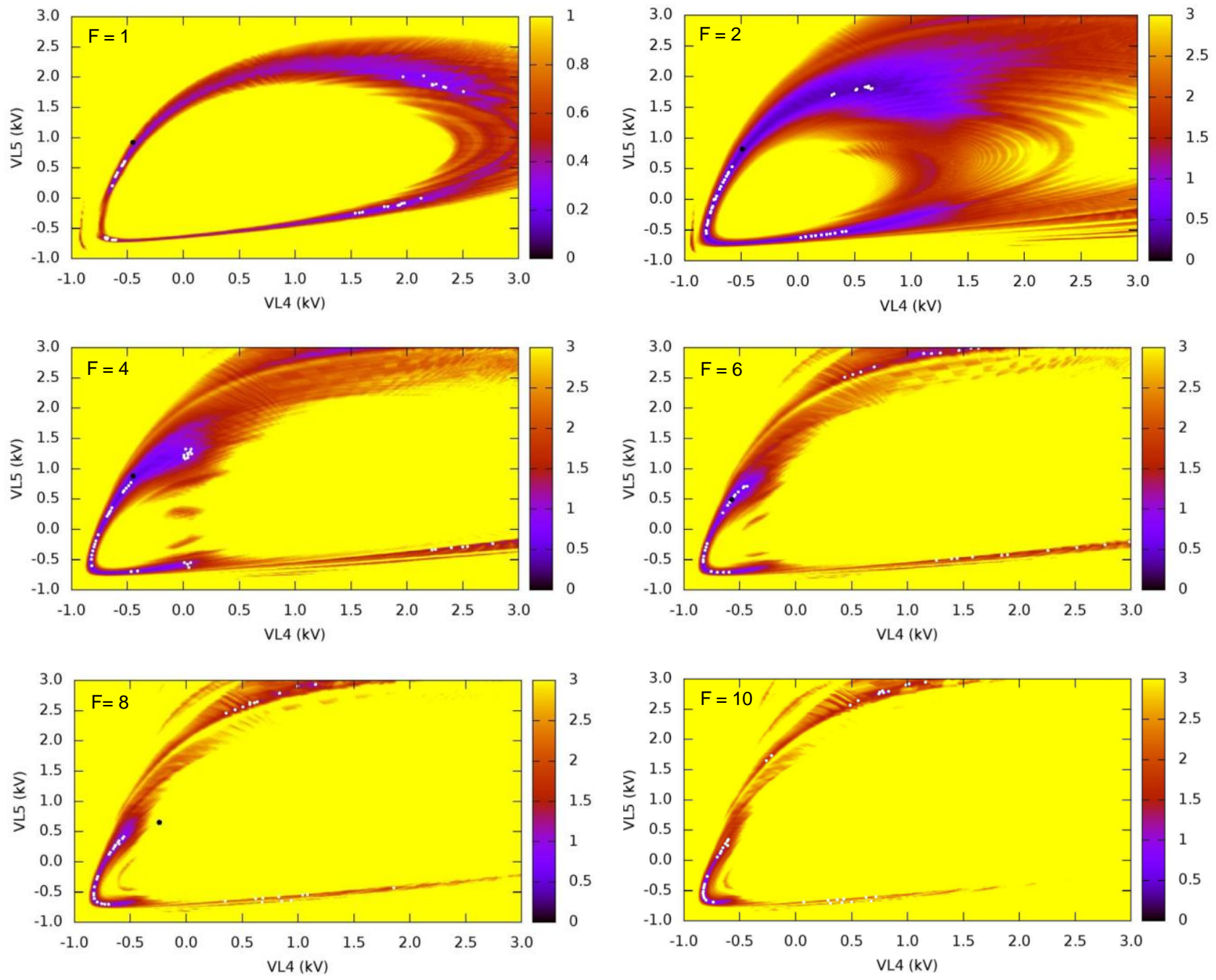

Figure 2. 2-D contours of the beam trace width $\Delta x_{\text {span }}$ versus VL4 and VL5 for different values of the pre-retardation factor $F$. Initial e- beam electron energy $E_{s 0}=1000 \mathrm{eV}$ and $\gamma=1.5$. Only values of $\Delta x_{s p a n}$ $\leq 3 \mathrm{~mm}$ are shown. White dots are some of the voltage combinations with the lowest $\Delta x_{\text {span }}$, while the black dots represent voltages that were experimentally determined in an empirical approach [3].

In Table 1 , it is interesting to note that the resolution $\mathcal{R}$ is seen to go through a minimum for $F=6$. It seems that for this particular set of lens voltages VLA and VL5, the spot size FWHM characterized by $\Delta x_{\mathrm{psd}}$ is smallest relative to other listed $F$ values. This is rather unexpected and could signal the onset of non-negligible aberration contributions for $F>6$. Typically, a strictly monotonically decreasing behaviour can be expected for $\Delta x_{\text {psd }}$ with increasing $F$ when aberrations are not considered [6]. 

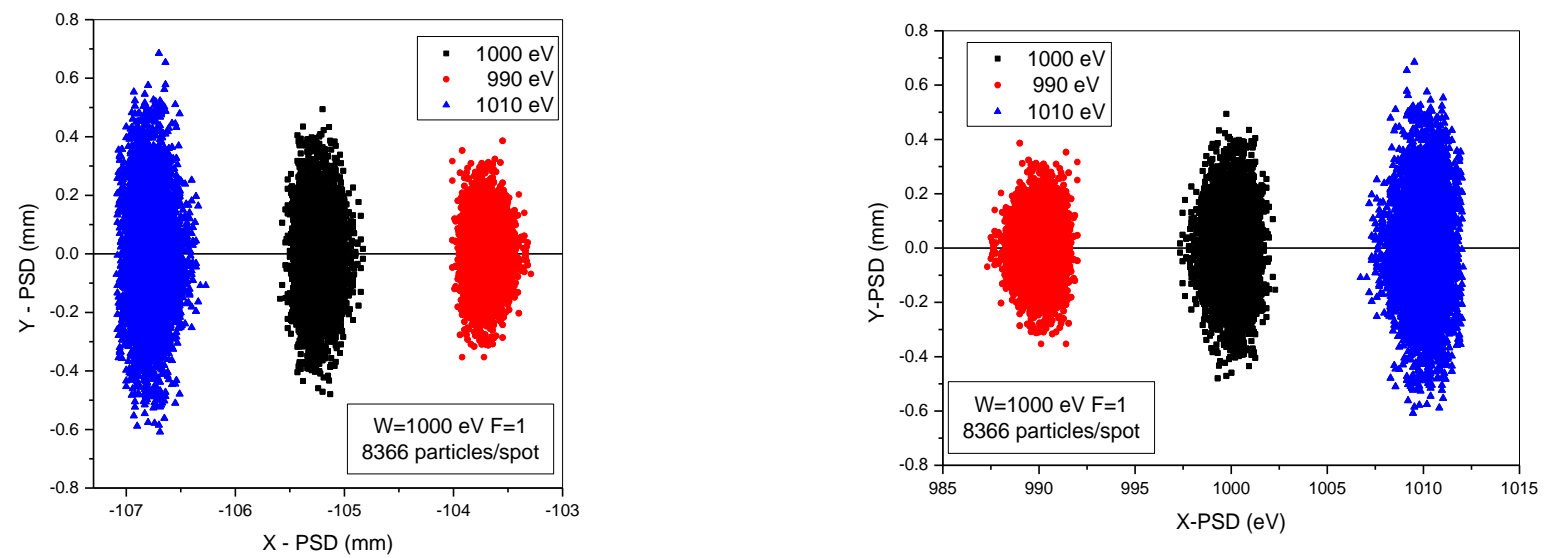

Figure 3. Simulation $(\mathrm{x}, \mathrm{y})$ spots obtained on the PSD for three electron beams with energies $E_{\mathrm{s} 0}=1000$ $\mathrm{eV}$ (black), $1010 \mathrm{eV}$ (blue) and $990 \mathrm{eV}$ (red). Left: $(x, y)$ coordinates on PSD plane, right: the data on the left have been energy calibrated. The $x$-axis lies along the energy dispersion direction of the HDA. Spectrometer voltages were tuned for a central reference energy of $1000 \mathrm{eV}$.

\begin{tabular}{cccccc}
\hline $\boldsymbol{F}$ & $\boldsymbol{V L 4}(\mathbf{V})$ & $\boldsymbol{V L 5}(\mathbf{V})$ & $\Delta x_{\mathrm{psd}}(\mathbf{m m})$ & $\boldsymbol{D}(\mathbf{m m})$ & $\mathcal{R}(\boldsymbol{\%})$ \\
\hline 1 & -697.2 & -655.9 & 0.315 & 153 & 0.206 \\
2 & -795.7 & -389.4 & 0.511 & 309 & 0.156 \\
4 & -530.7 & 637.3 & 0.598 & 630 & 0.095 \\
6 & -512.9 & 616.3 & 0.524 & 961 & 0.056 \\
8 & -826.8 & -522.5 & 0.871 & 1291 & 0.067 \\
\hline
\end{tabular}

Table 1. Optimal values of the lens voltages $V L 4$ and $V L 5$ giving minimum beam width as functions of the pre-retardation factor $F$ (for $E_{\mathrm{s} 0}=1000 \mathrm{eV}$ ). The corresponding values of the calculated dispersion and resolution are also listed.

In Figure 4 we plot and compare the 2-D and 3-D contour plots for both the experimental results shown on the left and the simulation results shown on the right on the same scale so they can be readily compared. The experimental data shown was taken using a "hot-wire" electron gun made from an ordinary flash light bulb used primarily for testing purposes and having no additional lens system or other control electrodes. While its intrinsic FWHM energy resolution can clearly be improved it was adequate for testing the lens voltages predicted by simulation in comparison to the older lens voltages used, which were found empirically from measurements with actual Auger lines [3]. A slight shift towards negative $y$-values in the PSD plane is observable, probably an indication of slight misalignment of the gun. The three experimental energy spots were accumulated independently, but are plotted here in the same figure to save space.

The $x$-direction is the HDA energy dispersion direction and peak projections along this axis when fitted by the appropriate response function (typically a Gaussian or Voigt profile) can provide the FWHM of the measured lines and provide important information about the energy resolution of the HDA, the effect of fringing fields, as well as contributions from higher order aberrations $[7,8]$. 

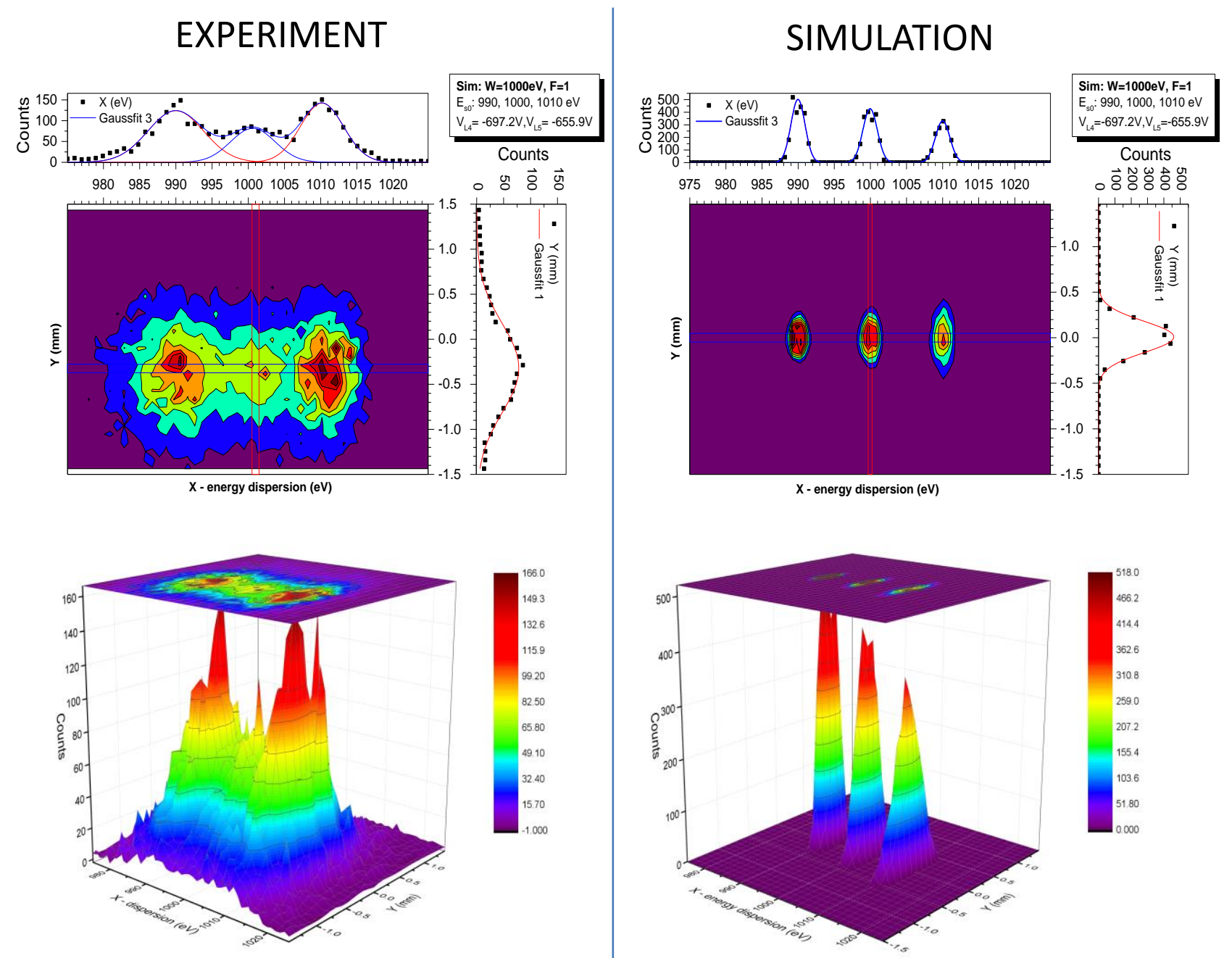

Figure 4. Comparison of simulation and experiment for $E_{s 0}=1000 \mathrm{eV}, F=1, V L 4=-697.2 \mathrm{~V}$ and $V L 5=-$ 655.9 V. Left: experimental results using an electron gun, right: simulation results (from data shown in Figure 3 right). Top: 2-D color contour plots with $\mathrm{X}$ and $\mathrm{Y}$ projections shown and fitted by Gaussians, bottom: 3-D color map surface with projection.

The $y$-direction, not usually discussed much in the literature, is of important diagnostic value since it can provide direct information about the focusing characteristics of the lens, as well as indicate any slight misalignments with respect to the optical axis as already mentioned above. The cylindrical symmetry of the lens should result in equal focusing of the beam in both the $x$ - and $y$-directions. However, in the $x$ direction this focusing is partly masked by the dispersion of the analyser making it more difficult to directly extract information about the lens. Alternatively, in the $y$-direction - because analyser influences are more limited - the focusing action of the lens is much clearer and directly observable and thus quantities such as the linear (and angular) magnification of the lens should be more readily extractable than from projections along the $x$-direction.

In summary, we have presented a theoretical method for finding the optimal working voltages on an HDA injection lens using CPO simulations and based on 1-D projections (beam trace width) 
minimization which is both fast and exhaustive and should lead to optimal energy resolution. These voltages are shown to lie on a family of elliptical-like contour curves of minimal trace width. We have also used some of the predicted lens voltage sets on the actual experimental apparatus and found them to be at least as good as or better than the previously experimentally determined ones.

This is a work in progress and we are still in the process of analysing and evaluating our results. Here we report only part of our most recent findings. A more detailed report will be presented as a full publication in the near future $[9,10]$.

References:

[1] http://apapes.physics.uoc.gr.

[2] SIMION 8.1, Scientific Instrument Services,Inc.,Ringoes, NJ, http://www.simion.com.

[3] EP Benis and TJM Zouros, J. Electron Spectrosc. 163 (2008), p. 28.

[4] TJM Zouros and EP Benis, J. Electron Spectrosc. 125 (2002) p. 221; erratum: ibid, 142 (2005), p. 175.

[5] EP Benis and TJM Zouros, Nucl. Instrum. Meth. Phys. Res. A 440 (2000), p. 462.

[6] TJM Zouros and EP Benis, Appl. Phys. Lett. 86 (2005), 094105.

[7] O Sise et al, J. Electron Spectrosc. 177 (2010), p. 42.

[8] M Dogan et al, Rev. Sci. Instrum. 84 (2013), 043105.

[9] G. Martínez et al, in preparation.

[10] We acknowledge partial support by the European Union (European Social Fund - ESF) and Greek national funds through the Operational Program "Education and Lifelong Learning" of the National Strategic Reference Framework (NSRF) Research Funding Program: THALES, Investing in knowledge society through the European Social Fund (Grant No. MIS 377289). This work was also supported by the Spanish Ministerio de Economía y Competitividad under project FIS2012-31230. 